\title{
Table analytique des matières.
}

A-Généralités . . . . . . . . . . . . . . . . . . . . 1

$1^{0}$ L'importance de la mesure . . . . . . . . . . . . . 1

$2^{\circ} \mathrm{La}$ précision demandée suivant le cas . . . . . . . . . 2

$3^{0} \mathrm{La}$ sensibilité des différents instruments de mesure . . . . 3

$4^{0}$ Signification des inscriptions portées sur les instruments . . 6

B - Mesures de l'intensité et de la tension . . . . . . . . . . . . . 9

$1^{0}$ Principes physiques . . . . . . . . . . . . . . . . . 9

$2^{0}$ Généralités sur la mesure d'intensité et de tension . . . . 11

$3^{0}$ Ampèremètres et voltmètres à cadre mobile pour courant continu . . . . . . . . . . . . . . . . . . . . 12

a) Construction et propriétés du système de mesure . . . . 12

b) Instruments de mesure industriels . . . . . . . . . . 13

c) Instruments de vérification, type «Z» . . . . . . . . . 17

d) Instruments de précision . . . . . . . . . . . . . 18

$4^{0}$ Ampèremètres et voltmètres électromagnétiques pour courant continu et alternatif... . . . . . . . . . . 23

a) Structure et propriétés du système de mesure . . . . . 23

b) Instruments de mesure industriels . . . . . . . . . . 24

$5^{0}$ Ampèremètres et voltmètres électrodynamiques pour courant alternatif . . . . . . . . . . . . . . . . 28

a) Structure et propriétés du système de mesure . . . . . 28

b) Instruments de précision . . . . . . . . . . . . . . . . . . 29

C - Mesures indirectes de l'intensité et de la tension . . . . . . 33

$1^{0}$ Généralités sur les mesures à l'aide de transformateurs de mesure . . . . . . . . . . . . . . . . . . . . . 33

a) Les différents types de transformateurs de mesure et leurs applications . . . . . . . . . . . . . . .

b) Règles générales de montage pour transformateurs de mesure . . . . . . . . . . . . . . . . . . . . 34

c) Calcul des constantes . . . . . . . . . . . . . . 40

$2^{0}$ Transformateurs d'intensité de construction normale . . . . 41

a) Remarques générales sur la construction . . . . . . . 41

b) Transformateurs d'intensité portatifs pour des tensions allant jusqu'à 650 volts . . . . . . . . . . . . . . . 44 
c) Transformateurs d'intensité de précision portatifs pour des tensions allant jusqu'a 15000 volts . . . . . . . 45

$3^{0}$ Transformateurs d'intensité à l'épreuve des courts-circuits . 49

a) Remarques générales . . . . . . . . . . . . . . 49

b) Transformateurs de traversée à barre primaire . . . . . 50

c) Transformateurs à deux traversées . . . . . . . . . 57

d) Transformateurs à tube transversal en porcelaine . . . . . $\quad 59$

$4^{0}$ Transformateurs de potentiel . . . . . . . . . . . . 62

a) Remarques générales concernant la structure . . . . . 62

b) Transformateurs de potentiel portatifs pour des tensions allant jusqu'à 600 volts . . . . . . . . . . . . . . 63

c) Transformateurs de potentiel portatifs pour des tensions allant jusqu'à 12000 volts . . . . . . . . . . . . 63

$5^{0}$ Emploi de traversées à condensateurs dans les mesures . . . $\quad 65$

a) Construction des traversées à condensateurs . . . . . 67

b) Méthodes de mesure . . . . . . . . . . . . . . . . . . 67

D - La mesure de très faibles intensités et tensions à l'aide de galvanomètres

$1^{0}$ Remarques générales concernant les diverses exécutions des galvanomètres . . . . . . . . . . . . . . . . . . . . . . . . . 69

20 Constante d'intensité et de tension . . . . . . . . . . 72

$3^{\circ}$ Détermination des constantes . . . . . . . . . . . . 74

$4^{\circ}$ Choix d'un galvanomètre destiné à une mesure déterminée 78

$5^{0}$ Réduction de la sensibilité . . . . . . . . . . . . . 78

$6^{0}$ Détermination des constantes de résistance . . . . . . . . 84

$7^{0}$ Mesures balistiques . . . . . . . . . . . . . . . . 85

$8^{0} \mathrm{La}$ constante balistique et sa détermination . . . . . . . . 87

$9^{\circ}$ Réduction de la sensibilité du galvanomètre balistique . . . 88

$10^{\circ}$ Mesure de petites intensités alternatives par l'emploi du thermocouple et du galvanomètre à courant continu . . . . 89

E - Mesure des puissances . . . . . , , . . . . . . . . 91

10 Remarques générales . . . . . . . . . . . . . . . . 91

a) Puissance active, réactive et apparente . . . . . . . 91

b) Direction de la puissance . . . . . . . . . . . . . . . . . 93

$2^{0}$ Les différents modèles des wattmètres . . . . . . . . . . . 96

a) Wattmètres électrodynamiques à circuit magnétique ouvert 96

b) Wattmètres électrodynamiques à circuit magnétique fermé 101

c) Wattmètres d'induction . . . . . . . . . . . . . . 106

$3^{0}$ Calcul des constantes pour les wattmètres portatifs . . . . 107

$4^{0}$ Schémas et règles de branchement . . . . . . . . . . . 109 
Table analytique des matières.

a) Mesures directe, semi-indirecte et indirecte . . . . . . 109

b) Règles de branchement pour wattmètres portatifs . . . 110

$5^{\circ}$ Schémas de branchement pour courant monophasé . . . . 113

$6^{0}$ Mesures en courant triphasé . . . . . . . . . . . . . 117

a) Méthodes de mesure pour la détermination de la puissance active . . . . . . . 117

b) Méthodes de mesure pour la détermination de la puissance réactive . . . . . . . . . . . . . . . . . . . . . . . . . .

c) Résistances additionnelles à commutateurs pour la mesure de la puissance active et réactive . . . . . . . . . 131

$7^{0}$ Schémas de branchement pour courant triphasé . . . . . 133

F - Etalonnage des ampèremètres, voltmètres et wattmètres au moyen de potentiomètres . . . . . . . . . . . . . . . 139

$1^{0}$ Principe de la mesure dite de «compensation» . . . . . . . 139

$2^{0}$ Montage intérieur du potentiomètre d’après Raps . . . . 141

$3^{0}$ Etalonnage des voltmètres . . . . . . . . . . . . . 116

$4^{0}$ Etalonnage des ampèremètres . . . . . . . . . . . . 150

$5^{0}$ Etalonnage d'un wattmètre . . . . . . . . . . . . . . 151

G - Etalonnage des compteurs d'électricité . . . . . . . . . 153

10 Remarques générales . . . . . . . . . . . . . . . . 153

$2^{0}$ Installations d'essai pour compteurs en courant continu . . . 154

$3^{0}$ Installation d'essai pour compteurs en courant alternatif . . 155

H - Essai des transformateurs de mesure . . . . . . . . . . 163

10 Essai des transformateurs d'intensité . . . . . . . . . . 163

$2^{0}$ Essai des transformateurs de potentiel . . . . . . . . . . 167

J- Mesure du facteur de puissance . . . . . . . . . . . . . . . . 169

10 Remarques générales . . . . . . . . . . . . . . . . 169

$2^{0}$ Système de mesure des phasemètres . . . . . . . . . . . 173

$3^{0}$ Schéma de branchement des phasemètres . . . . . . . . 175

K - Mesure de la fréquence et du nombre de tours . . . . . . . 177

$1^{0}$ Fréquencemètres . . . . . . . . . . . . . . . . . 177

$2^{\circ}$ Indicateur du nombre de tours sur place. . . . . . . . 181

$3^{0}$ Indicateur du nombre de tours à distance . . . . . . . . . 182

L - Détermination du sens des phases d'un secteur à courant alternatif . . . . . . . . . . . . . . . . . . . . . . . 185

M - Détermination du synchronisme . . . . . . . . . . . . 187

10 Remarques générales . . . . . . . . . . . . . . . . 187

$2^{0}$ Fréquencemètre double et voltmètre double . . . . . . . . 189

$3^{\circ}$ Voltmètre de zéro . . . . . . . . . . . . . $\quad . \quad . \quad . \quad 191$ 
40 Voltmètre à maximum Page

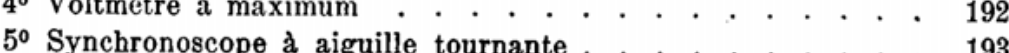

N - Mesure de la résistance d'un conducteur électrique . . . . 196

$1^{0}$ Mesure d'une résistance à l'aide d'un ampèremètre et d'un voltmètre . . . . . . . . . . . . . . . . . . . . . 196

a) Remarques générales . . . . . . . . . . . . . . . . . 196

b) Dispositif pour la détermination de faibles résistances . 197

c) Dispositif pour la détermination de la résistance d'un induit . . . . . . . . . . . . . . . . . . . . . 199

$2^{\circ}$ Mesure des résistances moyennes et élevées à l'aide du pont

de Wheatstone . . . . . . . . . . . . . . . . . 201

a) Remarques générales . . . . . . . . . . . . . . . . 201

b) Pont à fiches avec résistance de comporaison en série . . 205

c) Pont de précision avec résistances de comparaison en décades . . . . . . . . . . . . . . . . . . . 209

d) Pont de précision à manettes . . . . . . . . . . . . 213

e) Pont à fil pour courant alternatif . . . . . . . . . 215

$3^{\circ}$ Mesure de faibles résistances à l'aide du pont de Thomson . 216

a) Remarques générales . . . . . . . . . . . . . . 216

b) Ancien pont double de Thomson à fil calibré . . . . . 218

c) Pont de précision à doubles manettes . . . . . . . . 221

$4^{0}$ Ohmmètres à lecture directe . . . . . . . . . . . . . . 224

a) Remarques générales . . . . . . . . . . . . . . . . . 224

b) Ohmmètre à montage shunt . . . . . . . . . . . . . . . 225

c) Ohmmètre à montage série . . . . . . . . . . . . . . . . . 227

d) Ohmmètre basé sur le principe du logomètre . . . . . 228

O - Mesure des résistances d'isolement . . . . . . . . . . . . . 230

$1^{0}$ Prescriptions pour les mesures d'isolement . . . . . . . . 230

$2^{\circ}$ Remarques générales sur la construction des essayeurs d'isolement . . . . . . . . . . . . . . . . . . . 234

$3^{0}$ Essayeur d'isolement à cadre mobile et à magnéto . . . . 237

$4^{0}$ Mégohmmètre à cadres croisés et à magnéto . . . . . . . . . 239

a) Ancienne exécution à équipage à double cadre . . . . . 239

b) Nouveau mégohmmètre à triple cadre . . . . . . . . . . 240

$5^{0}$ Mesure de l'isolement d'une installation . . . . . . . . . 244

$\mathbf{P}$ - Mesure des résistances de terres . . . . . . . . . . . . . . . . 249

$1^{0}$ Remarques générales . . . . . . . . . . . . . . . . 249

$2^{\circ}$ Anciennes méthodes pour la mesure de la résistance des terres 251

a) Mesure à l'aide de deux prises de terre . . . . . . . 251

b) Mesure à l'aide d'une prise auxiliaire de valeur variable . 253

c) La méthode de Wiechert . . . . . . . . . . . . . 254 
Page

$3^{0}$ Pont à téléphone pour parafoudres . . . . . . . . . . 257

$4^{0}$ Le nouveau tellurohmmètre . . . . . . . . . . . . . 258

Q - Mesures des coefficients d'inductance . . . . . . . . . . . 263

$1^{0}$ Remarques générales. . . . . . . . . . . . . . . . 263

20 Pont pour la mesure de grands coefficients de self-inductance 268

$3^{0}$ Pont pour la mesure de petits coefficients de self-inductance 271

R - Mesures des capacités . . . . . . . . . . . . . . . $\underline{2} 72$

$1^{0}$ Remarques générales . . . . . . . . . . . . . . . . 272

$2^{\circ}$ Mesure à l'aide d'un galvanomètre balistique . . . . . . . 272

$3^{0}$ Mesure à l'aide du pont pour courant alternatif . . . . . 274

S - Recherche et localisation des défauts dans les conducteurs . . 275

$1^{0}$ Remarques générales. . . . . . . . . . . . . . . . 275

$2^{0}$ Recherche des défauts d'après la méthode de la boucle . . . 277

$3^{0}$ Recherche des défauts d'après la méthode de la chute de potentiel . . . . . . . . . . . . . . . . . 280

$4^{0}$ Recherche des défauts par des mesures de capacité . . . . 284

50 Pont à fil pour la recherche des défauts . . . . . . . . . . 285

T - Appareillage complet pour la recherche des défauts dans les câbles $\quad 287$

$1^{0}$ Appareil portatif de recherche des défauts dans les câbles haute et basse tension . . . . . . . . . . . . . . . 287

a) Montage intérieur de l'appareil . . . . . . . . . . . 287

b) Montage extérieur pour les différentes mesures . . . . 291

$2^{0}$ Dispositif portatif d'essai de câbles avec protection contre les courants parasites pour résistances d'isolement particulièrement élevées . . . . . . . . . . . . . . . . . . . . . . 294

a) Caractéristiques spéciales et connexions internes . . . . 294

b) Connexions externes pour des mesures d'isolement et de capacité . . . . . . . . . . . . . . . . . . . . 997

U - Protection par relais des réseaux de distribution . . . . 301

$1^{0}$ Remarques générales . . . . . . . . . . . . . . . . 301

$2^{\circ}$ Construction des différents relais . . . . . . . . . . 303

a) Relais à maximum d'intensité à action instantanée . . . 303

b) Relais temporisés à maximum d'intensité à retard fixe pour les fortes surcharges . . . . . . . . . . . . 305

c) Relais à maximum d'intensité à retard fixe indépendant de la surcharge . . . . . . . . . . . . . . . . 307

d) Relais électromagnétiques à maximum d'intensité . . . . 309 
Table analytique des matières.

e) Relais à retour d'énergie ou relais directionnels . . . . . 309

f) Relais auxiliaires . . . . . . . . . . . . . . . . . . . 313

$3^{\circ}$ Schémas de principe de la protection contre les surcharges . 315

a) Protection en cascade par relais temporisés à maximum d'intensité dans le cas d'un réseau radial à feeders simples . . . . . . . . . . . . . . . . . . . . 315

b) Protection des feeders où l'énergie s'écoule dans les deux sens . . . . . . . . . . . . . . . . . .

c) Protection contre les courts-circuits et les surcharges des feeders en parallèle à distribution d'énergie dans un seul sens, à l'aide de relais directionnels . . . . . . . . . . . . 321

d) Protection différentielle de deux feeders en parallèle contre les courts-circuits et les ruptures . . . . . . . .

e) Protection polygonale de plus de deux feeders en parallèle contre les courts-circuits, les ruptures et les défauts à la terre . . . . . . . . . . . . . . . . . . . . . 329

f) Protection des barres omnibus contre les courts-circuits

g) Protection différentielle des alternateurs et des transformateurs . . . . . . . . . . . . . . . . . . 338

$4^{0}$ Schémas de principe pour les indications des défauts à la terre 341

V -- Essais de rigidité diélectrique $\quad . \quad$. . . . . . . . . . . . . . . . . $\quad .347$

$1^{0}$ Pupitre d'essai à haute tension pour appareils électriques et instruments de mesure . . . . . . . . . . . . . . . . 347

$2^{0}$ Installation d'essai à haute tension pour courant alternatif 351

$3^{0}$ Installation d'essai pour courant continu sous haute tension 351

$4^{0}$ Installation d'essai des huiles pour transformateurs et disjoncteurs . . . . . . . . . . . . . . . . . 355

W - Essais des enroulements de machines . . . . . . . . . . 359

$1^{0}$ Remarques générales . . . . . . . . . . . . . . . . . 359

$2^{0}$ Montage et description de l'installation d'essai d'induits . . . 359

$3^{0}$ Recherche des courts-circuits dans les enroulements d'induits 361

$4^{0}$ Recherche des courts-circuits dans les différentes bobines . . 363

$5^{0}$ Recherche des défauts d'isolement . . . . . . . . . . . . . . 366

X - Détermination des propriétés magnétiques du fer . . . . . 367

$\mathbf{1}^{0}$ Remarques générales . . . . . . . . . . . . . . . . 367

$2^{0}$ Perméamètre de Koepsel , . . . . . . . . . . . . . . , . 369

$3^{\circ}$ L'appareil Epstein . . . . . . . . . . . . . . . . . 373

$4^{0} \mathrm{La}$ méthode différentielle . . . . . . . . . . . . . . . . 376 
Table analytique des matières.

XIII

Page

$\mathbf{Y}$ - Enregistrement et transmission à distance des valeurs de mesure . . . . . . . . . . . . . . . . . . . . . . 379

$1^{0}$ Enregistreurs à tracé continu . . . . . . . . . . . . . . . . . 379

a) Mode d'inscription des courbes . . . . . . . . . . . . . . . . 379

b) Exécution des appareils enregistreurs . . . . . . . . . . . 383

c) Commande synchronisée de plusieurs enregistreurs à encre 386

$2^{0}$ Appareils enregistreurs à tracé par points . . . . . . . . . . 389

$3^{0}$ Les oscillographes . . . . . . . . . . . . . . . . . . . . . 393

$4^{0}$ Mesures à distance. . . . . . . . . . . . . . . . . 403

Z - Appareils de réglage pour mesures électriques . . . . . . 407

$1^{0}$ Rhéostats pour le réglage de l'intensité et de la tension . . . 407

$2^{0}$ Rhéostats-transformateurs pour le réglage de l'intensité et de

la tension . . . . . . . . . . . . . . . . . . . 409

$3^{0}$ Transformateurs de phases et machines d'étalonnage . . . 411

$4^{0}$ Appareil de contrôle pour relais . . . . . . . . . . . . . 413

Appendlce . . . . . . . . . . . . . . . . . . . . . . . 416

Classification des appareils de mesure . . . . . . . . . . . . 416

Table de matières alphabétiques $\quad$. . . . . . . . . . . . . . . . . 427

Tableaux . . . . . . . . . . . . . . . . . . . . . . . 434

Autres publications de la maison Siemens . . . . . . . . . . . . . 141 
